\title{
Triple assessment as a preoperative diagnostic tool for breast cancer at Bugando Medical Centre in northwestern Tanzania
}

PHILLIPO L. CHALYA ${ }^{1 *}$, MONAFISHA K. LEMA ${ }^{2}$, JOSEPH B. MABULA ${ }^{1}$, PETER RAMBAU 3 , MABULA D. MCHEMBE $^{4}$, NESTORY MASALU ${ }^{5}$, GODFREY A. KASANGA ${ }^{6}$, GEOFREY GIITI ${ }^{1}$ and JAPHET M. GILYOMA ${ }^{1}$

${ }^{1}$ Department of Surgery, Catholic University of Health and Allied Sciences-Bugando, Mwanza, Tanzania

${ }^{2}$ Department of Surgery, Muhimbili National Hospital, Dar es Salaam, Tanzania

${ }^{3}$ Department of Pathology, Catholic University of Health and Allied Sciences-Bugando, Mwanza, Tanzania

${ }^{4}$ Department of Surgery, Muhimbili University of Health and Allied Sciences, Dar es Salaam, Tanzania

${ }^{5}$ Department of Oncology, Catholic University of Health and Allied Sciences-Bugando, Mwanza, Tanzania

${ }^{6}$ Department of Radiology, Catholic University of Health and Allied Sciences-Bugando, Mwanza, Tanzania

\begin{abstract}
The triple assessment in the pre-operative evaluation of breast cancer has been practiced in some hospitals in Tanzania. However, its validity and reliability has not been evaluated in any hospital in the country including the study area. This cross-sectional study was conducted at Bugando Medical Centre in northwestern Tanzania to determine the validity, reliability and applicability of triple assessment as an alternative to conventional open biopsy in the pre-operative diagnosis of breast cancer. A total of 212 female patients aged 35 years and above were studied. All patients underwent clinical evaluation, mammography and fine needle aspiration cytology. Histopathology was done to confirm the diagnosis. One hundred and twenty (56.6 \%) patients with a median age of 36 years had benign lesions and the remaining ninety-two (43.4\%) with a median age of 47 years had breast cancer $(P=0.002)$. With triple assessment, 92 patients were diagnostic for malignancy and 120 benign, respectively confirmed by histopathology. The Sensitivity, Specificity, Positive Predictive Value, Negative Predictive Value and Accuracy were $100.0 \%$. There were no false positive or false negative results. The Kappa statistic for the combination was 1.0 implying excellent agreement with histopathology. The majority of patients (59.9\%) underwent triple assessment on the same day with results being available to the patient within 24 to 72 hours. The triple assessment is an accurate and quick method for the evaluation of breast cancer and can be applied as a safe alternative for open biopsy when it is concordant..
\end{abstract}

Key words: Triple assessment, breast cancer, diagnosis, mammography, fine needle aspiration, Tanzania

\section{Introduction}

Breast cancer is the most common cancer and leading cause of cancer deaths among women worldwide (Abdallah et al, 1999; Christobel \& Baun, 2000). A breast lump is the most common symptom associated with both benign and malignant breast lesions (Khemka et al, 2009). With growing awareness in the general population, especially about breast cancer, a lump in the breast causes physical, emotional and psychological trauma to the patient and family members (Ahmed et al, 2007). Therefore, a distinction of benign from malignant is of paramount importance for patient and proper management (Abdallah et al, 1999; Ahmed et al, 2007).

A definitive diagnosis of benign lesion not only saves the patient from unnecessary physical, emotional and psychological trauma but also relieves the health services from undue burden. On the other hand, a definitive pre-operative diagnosis of malignant lesion provides many opportunities for patient's counseling and planning of possible single-stage surgical treatment (Brett et al, 1998; Abdallah et al, 1999; Ahmed et al, 2007). Any patient in cancer prone age with palpable breast abnormality must be thoroughly evaluated and investigated.

The first step in evaluation of breast lump is the clinical examination. Although sometimes a clinician can confidently make the diagnosis of benign or malignant lesion, the possibility of mistake is always there even in very experienced hands (Kaufmann et al, 1994; Brett et al, 1998; Abdallah et al, 1999; Butler et al, 2005; Klein, 2005; Ahmed et al, 2007).

\footnotetext{
*Correspondence: Dr. Phillipo L. Chalya; E-mail: drphillipoleo@yahoo.com
} 
Presently, a wide range of diagnosis modalities is available for the evaluation of breast lumps. This includes ultrasound and Doppler scanning, mammography and recently MRI and contrast enhanced Ultrasound (Michell, 1998; Steyaert, 2000). However, their cost and limited availability, not all patients can benefit from this.

Conventional open biopsy, considered as the gold standard for confirming the diagnosis, has significant morbidity, it is costly, invasive, time consuming and exposes the patient to unnecessary anxiety and more than one surgical procedure (Mande et al., 2004). It also risks escalating spread of malignancy and its lengthy time taken between the diagnostic procedure and acquisition of histopathological report can lead to delay in definitive diagnosis and treatment (Mande et al., 2004; Khemka et al., 2009). There is thus a need for developing a method for establishing the diagnosis pre-operatively, which is cost effective, least invasive and disturbing to the patient with accuracy comparable to open biopsy. The two techniques currently available that have excellent patient tolerability are mammography (Warwick, 1998; Graf et al., 2004) and fine needle aspiration cytology (Park \& Harm, 1997; Westened et al, 2001). However, if employed alone, the reliability of mammography and fine needle aspiration cytology is only around $82 \%$ and $78 \%$ respectively, which is less than satisfactory to be solely depended upon for the institution of definitive treatment (Afridi et al., 1995).

The triple assessment consisting of clinical evaluation, mammography and fine needle aspiration cytology has been routinely practiced in the developed world as an alternative to conventional open biopsy in the pre-operative diagnosis of breast lumps (Hught et al., 1998; Mande et al., 2004). It is simple, reliable, reproducible, less traumatic and cost effective in terms of money and time and its diagnostic accuracy has been reported to reach 100\% (Kaufmann et al., 1994; Mande et al., 2004). The triple assessment in the pre-operative evaluation of breast cancer has been practiced in some centres in Tanzania where mammography and fine needle aspiration cytology facilities are available. However, its validity and reliability has not been evaluated in any hospital in the country including the study area, and therefore its use as an alternative to conventional open biopsy in the pre-operative diagnosis of breast lumps is not justified. This existing knowledge gap prompted the authors to conduct this study in our centre to determine the validity and reliability of triple assessment and to assess whether it can be employed as an alternative to conventional biopsy in the pre-operative diagnosis of breast lumps at Bugando Medical Centre in north-western Tanzania.

\section{Methods and Patients}

\section{Study design and setting}

This was a cross-sectional study which was conducted in the surgical wards and at the surgical outpatient clinic of Bugando Medical Centre (BMC) between March 2009 and February 2012. Bugando Medical Centre is a tertiary care hospital located in Mwanza city in northwestern Tanzania and has a bed capacity of 1000. It is a referral, consultant and teaching hospital for the Catholic University of Health and Allied Sciences-Bugando (CUHAS-Bugando). BMC is one of the four largest referral hospitals in the country and serves as a referral centre for tertiary specialist care for a catchment population of approximately 13 million people from Mwanza, Mara, Kagera, Shinyanga, Tabora and Kigoma.

\section{Study subjects}

The study included all female patients aged 35 years and above who presented to the surgical outpatient clinic or in the surgical wards and consented for the study. Females below 35 years of age were excluded due to low sensitivity of the mammography in depiction of focal breast lesions resulting from glandular parenchyma. Patients with acute inflammatory signs, fungating masses, pregnant women and those with recurrent breast cancer on the same side were excluded from the study. Consecutive sampling was employed to select patients who fulfilled the 
inclusion criteria until the total number of patients needed for the study was reached. Kish and Leslie formula was used to estimate the minimum sample size. Recruitment of patients to participate in the study was done in the surgical wards and at the surgical outpatient clinic. Patients were screened for eligibility into the study and those who fulfilled the inclusion criteria were enrolled into the study. All patients enrolled into the study underwent routine clinical evaluation and then investigated by mammography and fine needle aspiration cytology.

Clinical evaluation included the patient's demographic details, reproductive history, history of breast diseases, and physical examination. Physical examination suggestive of malignant breast lump included hard, irregular lumps, nipple elevation and retraction, areola thickening, peau d'orange, skin tethering and erythema, axillary lymphadenopathy. Regular mobile lumps were considered benign. The clinical evaluation findings were recorded as clinical diagnosis.

Mammographs of patients were taken in the standard cranio-caudal and oblique views. The studies were reviewed and reported according to BIRADS protocol (Liberman \& Menell, 2002). The radiologist's report was recorded as radiological diagnosis. Fine needle aspiration cytology was aseptically taken from the lesion using $23 \mathrm{G}$ needle and $5 \mathrm{ml}$ syringe. Two slides of thin films was made, one fixed in $95 \%$ alcohol for PAP stain and one air dried for MGG stain. The pathologist's report was recorded as cytological diagnosis. The clinical, mammography and Fine needle aspiration cytology diagnoses were respectively being recorded as benign, suspicious or malignancy. Suspicious cases were those, which fell in BIRADS IV category on mammography and showed atypical cells without frank malignancy on fine needle aspiration cytology. In this study, all suspicious cases were regarded as malignant. Histopathological report of biopsies taken from specimens removed after therapeutic or definitive surgery was corded as benign or malignant.

The triple assessment was scored as concordant if the three elements have either all malignant or all benign results. In this study, all malignant results were regarded as true positive and all benign results were regarded as true negative. It was scored as disconcordant if the three elements have neither all malignant nor all benign results. Definitive treatment started when the three diagnostic procedure concur (i.e. concordant score). In case of diagnostic discrepancy (i.e. disconcordant score), open biopsy was done. The subsequent modifications in treatment were guided by histopathological results.

\section{Statistical data analysis}

Statistical data analysis was done using SPSS statistical software version 15.0 (SPSS, Inc, Chicago, IL). Variables were cross-tabulated against histology, the gold standard. The sensitivity, specificity, Positive Predictive Value (PPV) and Negative Predictive Value (NPV), accuracy and error were determined for the individual methods (i.e. clinical, mammography and Fine needle aspiration cytology) and when combined (i.e. triple assessment). A p-value obtained for each observation was considered significant if found less than 0.05.The Kappa statistic was calculated for each individual methods and their combination to determine the degree of agreement with histology. A value above 0.75 suggested excellent agreements with histopathology, 0.40 to 0.75 is intermediate to good agreement and below 0.40 suggested poor agreement.

\section{Ethical considerations}

Ethical approval to conduct the study was obtained from the CUHAS-Bugando/BMC joint institutional ethic review committee before the commencement of the study. Informed consent was sought from each patient before being enrolled into the study.

\section{Results}

During the period under review, a total of 212 patients with breast lumps were enrolled in the study. Their ages ranged from 35 to 78 years with a median age of 45 years. One hundred and 
twenty $(56.6 \%)$ patients with a median age of 36 years had benign lesions and remaining ninetytwo (43.4\%) patients with a median age of 47 years had malignant lesions $(P=0.002)$. A total of $182(85.8 \%)$ patients were in good general condition - Kanorfsky scale above 70\% $(P=0.000)$. On clinical evaluation, 107 patients were diagnosed with malignant lesions of who 82 was True Positive. Ninety-five of the 105 diagnosed have benign lesions were True Negative $(P=0.012)$. The False positive and False negative were $10.9 \%$ and $20.8 \%$ respectively (Table 1 ).

Table 1: Clinical diagnosis, mammography, fine needle aspiration cytology and triple assessment versus histopathological examination

\begin{tabular}{|c|c|c|c|c|c|c|}
\hline \multirow[t]{2}{*}{ Type of diagnosis } & \multirow[t]{2}{*}{ Type of lesion } & \multicolumn{3}{|c|}{ Histopathology } & \multirow[t]{2}{*}{$x^{2}$} & \multirow[t]{2}{*}{ P-value } \\
\hline & & Malignant & Benign & Total & & \\
\hline \multirow[t]{3}{*}{ Clinical diagnosis } & Malignant & 82 & 25 & 107 & 198.2 & 0.012 \\
\hline & Benign & 10 & 95 & 105 & & \\
\hline & Total & 92 & 120 & 212 & & \\
\hline \multirow[t]{3}{*}{ Mammography } & Malignant & 83 & 22 & 105 & 56.8 & 0.002 \\
\hline & Benign & 9 & 98 & 107 & & \\
\hline & Total & 92 & 120 & 212 & & \\
\hline \multirow[t]{3}{*}{ Fine needle aspiration cytology } & Malignant & 87 & 23 & 110 & 71.7 & 0.000 \\
\hline & Benign & 5 & 97 & 102 & & \\
\hline & Total & 92 & 120 & 212 & & \\
\hline \multirow[t]{3}{*}{ Triple assessment } & Malignant & 92 & 0 & 92 & 89.1 & 0.001 \\
\hline & Benign & 0 & 120 & 120 & & \\
\hline & Total & 92 & 120 & 212 & & \\
\hline
\end{tabular}

From mammography, 83 patients out 105 were True Positive and 98 patients out of 107 were True Negative $(P=0.002)$. The False positive and False negative were $9.8 \%$ and $18.3 \%$ respectively. From FNAC, 87 out of 110 patients were True Positive. 97 patients out of 102 were True Negative $(P=0.000)$. The False positive and False negative were $5.4 \%$ and $19.2 \%$ respectively. In the triple assessment, 92 and 120 patients were respectively confirmed by histopathology to have malignancy and benign disease $(P=0.001)$. There were no False positive or False negative results. One hundred and twenty seven (59.9\%) patients underwent Triple assessment on the same day with results being available to the patient within 24 to 72 hours. The sensitivity, specificity, Positive Predictive Value, Negative Predictive Value, accuracy, error and Kappa statistics are shown in Table 2.

Table 2: Analysis of the Triple assessment and its components for detection of breast cancer

\begin{tabular}{llllllll}
\hline $\begin{array}{l}\text { Diagnostic } \\
\text { method }\end{array}$ & Sensitivity & Specificity & PPV & NPV & Accuracy & Error & Kappa statistic \\
\hline $\begin{array}{l}\text { Clinical } \\
\text { diagnosis }\end{array}$ & 89.1 & 79.2 & 76.6 & 90.5 & 83.5 & 16.5 & 0.68 \\
$\begin{array}{l}\text { Mammography } \\
\text { FNAC }\end{array}$ & 90.2 & 81.7 & 79.0 & 91.6 & 85.4 & 14.6 & 0.74 \\
$\begin{array}{l}\text { Triple } \\
\text { assessment }\end{array}$ & 100 & 79.1 & 80.8 & 97.4 & 86.8 & 13.2 & 0.76 \\
\hline
\end{tabular}

\section{Discussion}

Breast cancer is the commonest malignancy in females and a leading cause of cancer related deaths worldwide (Abdallah et al, 1999; Christobel \& Baun, 2000). In this review, the incidence of breast cancer presenting as lump was $43.4 \%$ which is comparable with other studies (Ahmed et al, 2007; Khemka et al, 2009). High incidence of 67.0\% was reported by Mande et al (2004) in Uganda. We could not find in literature the reasons for this difference. 
In this study, patients with benign lesions were found to be younger than patients with breast cancer which is in agreement with other studies (Mande et al, 2004; Ahmed et al, 2007; Khemka et al, 2009). The median age at diagnosis in patients with breast cancer in our study was 47 years, which is lower than what is seen in Western countries (Parkin et al., 2002). Few studies have shown that breast cancer in Africans tends to present at a young age compared to women in Western countries (Ikpatt et al., 2002; Fregene et al., 2005). The reason for this observation is not clear.

Clinical evaluation has been reported to be an essential first step in the pre-operative diagnosis of breast cancer (Kaufmann et al, 1994; Brett et al, 1998; Abdallah et al, 1999; Mande et al., 2004; Butler et al, 2005; Klein, 2005; Ahmed et al, 2007). In this study, the diagnostic accuracy of clinical evaluation was $83.5 \%$ with Sensitivity, Specificity, PPV and NPV of $89.1 \%, 79.2 \%, 76.6 \%$ and $90.5 \%$ respectively. The diagnostic error was high (16.5\%). There was good agreement with histopathology $(k=0.68)$. These findings are comparable with findings of other studies (Mande et al., 2004; Ahmed et al, 2007). Because of high diagnostic error as seen in this study, the clinical diagnosis alone is not reliable and therefore it does not warrant proceeding to definitive treatment. The high diagnostic accuracy of clinical diagnosis in the present study can be attributed to the fact that the majority of patients presented late with advanced disease and therefore the diagnosis of breast cancer was straightforward. False positive diagnoses in this study may be resulted from the high index of suspicion and prudence towards breast lumps and False negative clinical diagnoses may be due to atypical presentations of some breast lumps.

Mammography is an essential component in the pre-operative assessment of breast cancer. It serves to characterize and determine the extent of the mass and to evaluate the breasts for clinically occult lesions (Graf et al., 2004). The sensitivity of diagnostic mammography has been reported in most studies to be around $90 \%$ and the specificity up to $88 \%$ (Barlow et al., 2002). Therefore, mammography alone is not reliable to allow for the institution of definitive treatment. The known false-negative rate of mammography is between $8 \%$ and $10 \%$ (D'Orsi et al., 1994; Masroor et al., 2009). The sensitivity of mammography is decreased by dense breast tissue obscuring a lesion. Breast ultrasound has been found to be a useful adjunct to mammography in women with dense breast by detecting small cancers not identified on mammography or clinical breast examination (Masroor et al., 2009). False-negative results arise with poor technique and inadequate views that do not include the mass, or when the findings are misinterpreted by the radiologist, notably when there is overlap in the mammographic features of benign and malignant masses (Masroor et al., 2009). Our mammographic findings in this study demonstrated high Sensitivity and Accuracy which is consistent with findings in other studies (Mande et al., 2004; Ahmed et al, 2007). This observation can be explained by the fact that all the mammographic examinations were preformed by experienced radiologists, hence, decreasing the diagnostic error. False negative in this study may be resulted from soft, cystic or ill-defined breast lumps, and lumps close to chest wall. However, because of high False negative result in this study, mammography if employed alone, is less than satisfactory to be solely depended upon for the institution of definitive treatment.

Fine needle aspiration cytology (FNAC) of breast lump is an accepted and established method to determine the nature of the lump and it may play an important role when it is difficult to determine the nature of breast lump by clinical examination. It has been shown that, FNAC can reduce the number of open breast biopsies (Hindle et al., 1993). FNAC has been found to have sensitivity ranging from $82 \%$ to $97.5 \%$ and specificity of more than $99 \%$ (Yong et al., 1999; Dutta et al., 2001). In this study, sensitivity, specificity and accuracy of breast FNAC in diagnosis of malignancy was $94.6 \%, 80.8 \%$ and $86.8 \%$ respectively, which was quite comparable to the findings of the other studies (Mande et al., 2004; Ahmed et al, 2007). Studies have shown that FNAC has some limitations that tumor architecture is less described by FNAC while vascular invasion and tumor size cannot be accurately described by small sample and because of this, many surgeons are reluctant to consider positive cytology results as the only criterion for performing definitive 
surgery since no distinction is possible between infiltrating and non-infiltrating lesions (Tiwari, 2007). In the present study, False positive results were very minimal compared to False negative results (5.4\% versus $19.2 \%$ ). False negative and inconclusive reports were commonly resulted from acellular aspirates from very hard lumps, hemorrhagic aspirates from highly vascular tumors and aspirates from cystic lumps. Another reason for the high false negative rate in our study could be due to sampling error. This can be minimized by proper localization and aspiration technique (McManus \& Anderson, 2001). Proper localization can be aided using ultrasound. In order to reduce false negative as much as possible and in order to get high accuracy of FNAC is limited by the skill of the surgeon in obtaining the specimen (ensure that appropriate area is biopsed) and by the experience of cytopathologist in its interpretation (Nicholson et al., 1998). High False negative rate in this study limits FNAC, when applied alone, to be solely depended upon for the institution of definitive treatment.

The triple assessment, consisting of clinical evaluation, mammography and fine needle aspiration cytology has been recommended as a gold standard diagnostic tool in the preoperative diagnosis of breast cancer and can be used as an alternative to conventional open biopsy (Hught et al, 1998; Mande et al, 2004). When the three assessments are performed adequately and produce concordant results, the triple assessment diagnostic accuracy approaches $100 \%$ and definitive treatment can be started before histopathology (Hught et al, 1998; Mande et al, 2004). Disconcordant results or results that cannot be evaluated may indicate the need for open biopsy (Kaufmann et al, 1994; Veto et al., 1995; Robertson \& Evans, 1997; Mande et al, 2004). Therefore, triple assessment can reduce anxiety and unnecessary surgical procedures as well as delay in diagnosis. In agreement with other studies (Hught et al, 1998; Mande et al, 2004), the triple assessment in this study demonstrated 100\% Sensitivity, Specificity, Positive Predictive Value and Negative Predictive Value respectively and Accuracy was $100.0 \%$. Error was $0.0 \%$. The Kappa statistic for the combination was 1.0 implying excellent agreement with histology. The high Sensitivity, Specificity and diagnostic accuracy of triple assessment obtained in our series of palpable breast lumps implies that the assessments were performed adequately by a well trained and experienced team comprising of surgeons, radiologists and pathologists (cytologists). In this study, more than fifty percent of patients underwent Triple assessment on the same day with results being available to the patient within 24 to 72 hours, this decreased the time required for definite treatment, as well as relieved the anxiety of the patients. The triple assessment is therefore cost effective, easy to perform and time saving approach, however, it can be applied only in those institutions where excellent imaging facilities as well as services of a cytopathologist are available. Furthermore, it needs absolute collaboration between surgeons, radiologist and pathologist. Irregular availability of mammography (due to breakdown or inability of patients to afford) was the major limitation of this study. However, despite this limitation, the study has shown that the triple assessment is reproducible, making it a valid and reliable diagnostic tool for the pre-operative diagnosis of breast cancer.

In conclusion, the triple assessment is an accurate, simple and cost-effective method for the evaluation of breast cancer and can be applied at our centre as a safe alternative for open biopsy when it is concordant and open biopsy should be reserved only for disconcordant results. It is recommended that the triple assessment should be practiced at our centre as a gold standard diagnostic tool for breast cancer and definitive treatment can safely be started before histopathology if the triple assessment results show concordance. This will subsequently reduce the number of open breast biopsies significantly.

\section{Acknowledgements}

The authors would to acknowledge all those who were involved in the care of our study patients and those who provided support in the preparation of this manuscript. 


\section{References}

Abdullah, P., Malik, A., Zahir, N., Zahur-ur-Rehman, Abdus S. \& Mehmood A. (1999) Breast lumpswhat they actually represent. Journal of College of Physicians and Surgeons Pakistan 9, 4648.

Ahmed, I., Nazir, R., Chaudhary, M.Y. \& Kundi, S. (2007) Triple assessment of breast lump. Journal of College of Physicians and Surgeons Pakistan 17, 535-538.

Afridi, S., Malik, K. \& Waheed, I. (1995) Role of fine needle aspiration biopsy and cytology (FNABC) in breast lumps. Journal of College of Physicians and Surgeons Pakistan 5, 75-77.

Barlow, W.E., Lehman, C.D., Zheng, Y., Ballard-Barbash, R., Yankaskas, B.C. \& Cutter, G.R. (2002) Performance of diagnostic mammography for women with signs or symptoms of breast cancer. Journal of National Cancer Institute 2002, 94, 1151-1159.

Brett, J., Austoker, J. \& Ong, G. (1998) Do women who undergo further investigation for breast screening suffer adverse psychological consequences? A multi-center follow-up study comparing different breast screening result groups five months after their last breast screening appointment. Public Health Medicine 20, 396-403.

Butler, J.A., Vargas, H.I. \& Wilson, S.E. (1990) Accuracy of combined clinical mammographicytologic diagnosis of dominant breast masses . A prospective study. Archieve of Surgery 125, 893-895.

Christobel, M.S. \& Baun, M. (2000) The breast. In: Russel RCG, Williams NS, Bulstoode, CJK, (edi). Bailey and Love's Short Practice Of Surgery. 23rd ed. London: Arnold, 749-72.

D’Orsi, C.J., Adler, D.D. \& Ikeda, D.M. (1994) Breast imaging. Radiology 190, 936.

Dutta, S.K., Chattopadhyaya, A. \& Roy, S. (2001) Fine needle aspiration and imprint cytology in the diagnosis of breast lesions. Journal of the Indian Medical Association 99, 421-423.

Fregene, A. \& Newman, L.A. (2005) Breast cancer in sub-Saharan Africa: how does it relate to a breast cancer in African-American women? Cancer 103, 1540-1550.

Graf, O., Helbich, T.H., Fuchsjaeger, M.H., Hopf, G., Morgun, M. \& Graf, C. (2004) Follow-up of palpable circumscribed non-calcified solid breast masses at mammography and US: can biopsy be averted? Radiology 233, 850-856.

Hindle, W.H., Payne, P.A. \& Pan, E.Y. (1993) The use of fine- needle aspiration in the evaluation of persistent palpable dominant breast masses. American Journal of Obstetrics and Gynaecology 168, 1814-1818.

Hughes, J.E., Royle, G.T., Buchanan, R. \& Taylor, I. (1986) Depression and social stress among patients with benign breast disease. British Journal of Surgery 73, 997-999.

Hught, T.S., Trudeau, M.E. \& Reyd, C.D. (1998) The diagnostic accuracy of fine needle aspiration cytology, physical examination and mammography in the diagnosis of palpable breast lumps. Cancer Journal of Surgery 42, 8-9.

Ikpatt, O.F., Kuopio, T., Ndoma-Egba, R. \& Collan, Y. (2002) Breast cancer in Nigeria and Finland: epidemiological, clinical and histological comparison. Anticancer Research 22, 3005-3015.

Kaufmann, Z., Shiptz, B., Shapiro, M., Rona, R., Lew, S. \& Dinbar, A. (1994) Triple approach in the diagnosis of dominant breast masses: combined physical examination, mammography and fine needle aspiration. Journal of Surgical Oncology 56, 254-257.

Khemka, A., Chakrabarti, N., Shah, S. \& Patel, V. (2009) Palpable breast lumps: Fine needle aspiration cytology versus Histopathology: a correlation of diagnostic accuracy. The Internet Journal of Surgery 18:2

Klein, S. (2005) Evaluation of palpable breast masses. American Family Physician 71, 1731-1738.

Liberman, L. \& Menell, J.H. (2002) Breast imaging reporting and data system (BIRADS). Radiologic Clinics of North America 40, 409-430.

Mande, N., Gakwaya, A.M., Byanyima, R.K., Othieno, F. (2004) The triple assessment in the preoperative evaluation of patients with breast cancer in Mulago Hospital, Kampala- Uganda. East and Central African Journal of Surgery 9, 111-116 
Masroor, I., Ahmed, MN., Pasha, S. (2009) To evaluate the role of sonography as an adjunct to mammography $\mathrm{n}$ women with dense breasts. Journal of Pakistan Medical Association 59, 298-301.

McManus, DT. \& Anderson, N.H. (2001) Fine needle aspiration cytology of the breast. Current Diagnostic Pathology 7, 262-271.

Michell, M.J. (1998) The breast. In: David S. Textbook of Radiology and Imaging. 6th ed. London: Churchill Livingstone, 1429-1460.

Nicholson, S., Sainsbury, J.R.C., Wadehra, V. \& Needham G.K. (1988) Use of FNAC with immediate reporting in the diagnosis of breast disease. British Journal of Surgery 75, 847-850.

Parkin, D.M., Bray, F.I., Ferlay, J. \& Pissani, P. (2002) Global cancer statistics 2002. Journal of Clinical Cancer 55, 74-108.

Park, I.A. \& Ham, E.K. (1997) FNAC of palpable breast lesions. Histologic subtype in false negatives cases. Acta Cytologica 41,1131-1138.

Robinson, I.A., McKee, G., Nicholson, A., D’Arcy, J., Jackson, P.A. \& Cook, M.G. (1994) Prognostic value of cytological grading of fine-needle aspirates from breast carcinomas. Lancet 343, 947-949.

Steyaert, L. (2000) Doppler sonography in breast pathology. Journal Belge de Radiologie - Belgisch Tijdschrift voor Radiologi 83, 121-122.

Tiwari, M. (2007) Role of fine needle aspiration cytology in diagnosis of breast lumps. Kathmandu University Medical Journal 5, 215-217.

Veto, J., Pommier, R., Schmidt, W. (1995) Use of triple test for palpable breast lesions yields high diagnostic accuracy and cost savings. American Journal of Surgery 169, 519-522.

Warwick, D.J. (1988) Ultrasound and mammography in management of breast cancer. British Journal of Surgery 75, 243-245.

Westened, P.J., Sever, A.R., Beekman-De Volder, H.J. \& Liem, S.J. (2001) A comparison of aspiration cytology and core needle biopsy in the evaluation of breast lesions. Cancer 93, 146-150.

Yong, W.S., Chia, K.H., Poh, W.T. \& Wong, O.Y. (1999) A comparison of trucut biopsy with fine needle aspiration cytology in the diagnosis of breast cancer. Singapore Medical Journal 40, 587-589. 